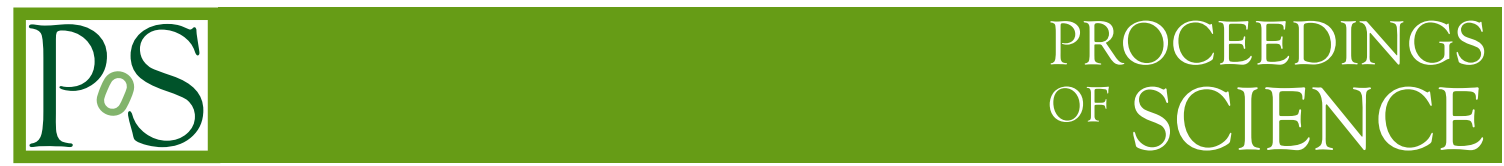

\title{
Search for vector-like quarks with the ATLAS Detector
}

\author{
Allison McCarn Deiana* \\ On behalf of the ATLAS Collaboration \\ Southern Methodist University \\ E-mail: adeiana@smu.edu
}

Vector-like quarks appear in many theories beyond the Standard Model as a way to cancel the mass divergence for the Higgs boson. The current status of the ATLAS searches for the production of vector-like quarks is reviewed for proton-proton collisions with $\sqrt{s}=13 \mathrm{TeV}$ produced by the Large Hadron Collider. The results and the complementarity of the various searches are discussed.

The 39th International Conference on High Energy Physics (ICHEP2018)

4-11 July, 2018

Seoul, Korea

${ }^{*}$ Speaker. 


\section{Introduction}

Vector-like quarks (VLQs) are predicted by many theories beyond the Standard Model, e.g. composite Higgs, which are designed to address the divergence of the Higgs boson mass. VLQs are spin-1/2 particles, but are 'vector-like' in the sense that they have left- and right-handed components with the same color and electroweak quantum numbers. VLQs can arise in singlets, doublets, or triplets, and can have a charge of $2 / 3,-1 / 3,5 / 3$ or $-4 / 3$ [2]. For this summary of current ATLAS results, only VLQs in singlets or doublets with a charge of $2 / 3(T)$ or $-1 / 3(B)$ are considered, and only decay modes to a top or bottom quark and a boson, under the assumption that $\mathrm{BR}(T \rightarrow H t)+$ $\mathrm{BR}(T \rightarrow Z t)+\mathrm{BR}(T \rightarrow W b)=1$ and $\mathrm{BR}(B \rightarrow H b)+\mathrm{BR}(B \rightarrow Z b)+\mathrm{BR}(B \rightarrow W t)=1$.

VLQs can be produced in pairs or singly. The search for pair-produced VLQs is relatively model independent, since the production cross section depends only on the VLQ mass. On the other hand, singly-produced VLQs can have higher production cross sections at larger VLQ mass for some models. The status of searches with the ATLAS detector [1] at the LHC for pair-production of VLQs is described in Sec. 2, and the status for single-production is described in Sec. 3.

\section{Pair production of vector-like quarks}

The ATLAS Collaboration has searched for pair-produced VLQs through analyses targeting particular final states, and has then combined the results of these channels. All of the current results for pair-production use $36 \mathrm{fb}^{-1}$ of $p p$ collision data at $\sqrt{s}=13 \mathrm{TeV}$ recorded by the ATLAS detector.

Earlier searches for VLQ published in 2017-2018 include searches for $T T \rightarrow H t+X$ [3], $T T \rightarrow W b+X$ [4], BB $\rightarrow W t+X$ [5] and $T T \rightarrow Z t+X$ with $Z$ decaying to neutrinos [6]. where $X$ refers to any possible considered VLQ decay mode. Multiple new searches for pair production were shown for the first time at this conference, and they include $T T / B B \rightarrow Z t / b+Z$ with $Z$ decaying to leptons [7], the search for $T T$ or $B B$ in same-sign dilepton or trilepton final states [8], the search for $T T$ or $B B$ in fully hadronic states [9], and the combination of all listed ATLAS results [10].

This is the first full combination for VLQ results at the ATLAS collaboration, and it required all participating channels to carefully maintain orthogonality in their signal selections. The combination benefits from the complementarity of the various searches. Each search is evaluated for the entire plane of possible branching ratios, but each is particularly strong in the branching ratios that correspond most closely to their targeted final state. For instance, the case where $\operatorname{BR}(B \rightarrow H b)$ is near $100 \%$ is strengthened by the fully-hadronic search, since it is difficult to probe with leptonic final states. The combined limit on the pair-production of VLQs is significantly stronger than any analysis alone, and it extends the mass exclusion to 1.31 (1.03) TeV for $T(B)$ VLQs with any considered combination of branching ratios, as seen in Fig. 1. In terms of specific models, the branching ratios for $\mathrm{SU}(2)$ singlets and doublets are indicated on each plane.

\section{Single production of vector-like quarks}

The searches for singly-produced VLQs in $p p$ collision data with $\sqrt{s}=13 \mathrm{TeV}$ recorded by the ATLAS detector include $T \rightarrow W b$ (using $3.2 \mathrm{fb}^{-1}$ of data), $T / B \rightarrow Z t / b$ (using $36 \mathrm{fb}^{-1}$ of 

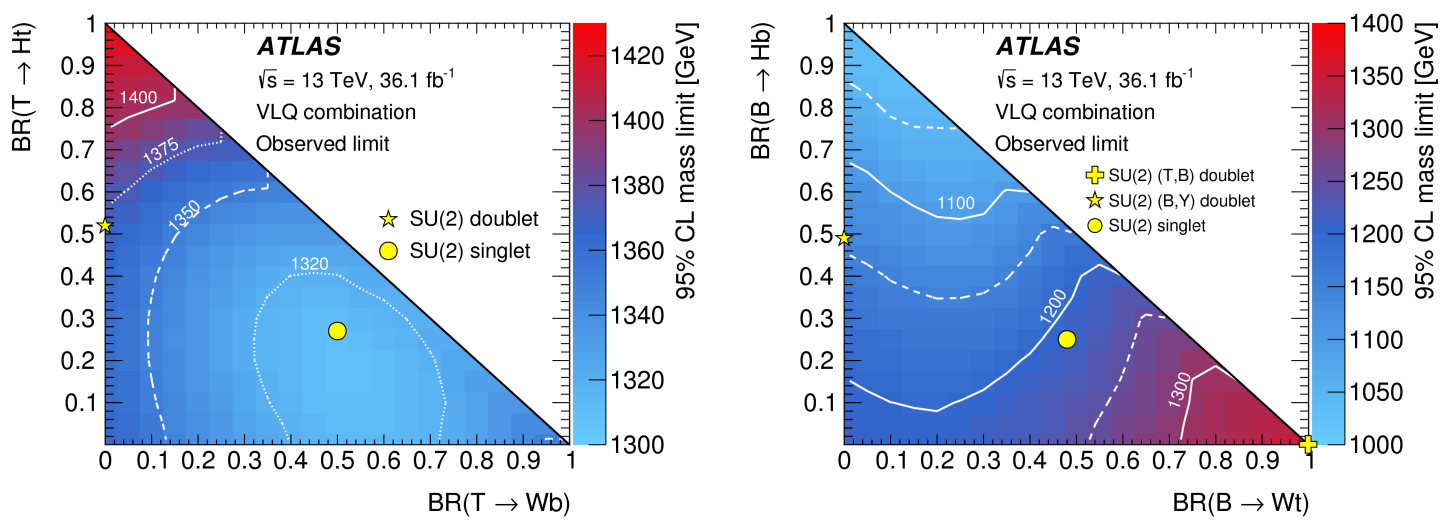

Figure 1: Observed lower limits at 95\% Confidence Level on the mass of the (left) $T$ and (right) $B$ as a function of branching ratio, for the combination of all ATLAS searches for pair-produced vector-like quarks that are included in this presentation. These limits assume $\mathrm{BR}(T \rightarrow H t)+\mathrm{BR}(T \rightarrow Z t)+\mathrm{BR}(T \rightarrow W b)=$ 1 and $\mathrm{BR}(B \rightarrow H b)+\mathrm{BR}(B \rightarrow Z b)+\mathrm{BR}(B \rightarrow W t)=1$. The yellow markers indicate the branching ratios for the SU(2) singlet and doublet scenarios with masses above $800 \mathrm{GeV}$ for which they are approximately independent of the VLQ mass. Figures are from Ref. [10].

data), and $B \rightarrow H b$ with $H$ decaying to two photons (using $80 \mathrm{fb}^{-1}$ of data). The production cross sections for these searches are model-dependent, so assumptions must be made in order to interpret the upper limits in terms of a physical model. The latest results, shown in Fig. 2, consider the case of a generalized coupling $\kappa_{T}$ or $\kappa_{B}$, which is set to a value of $0.5[13,14]$.
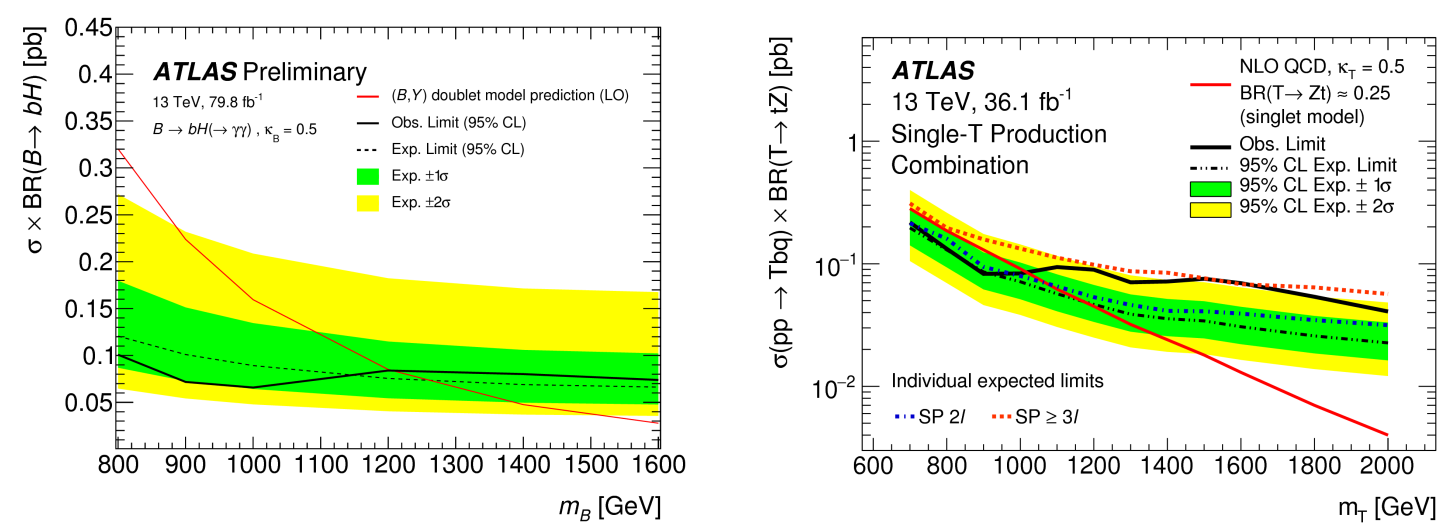

Figure 2: Upper limits at 95\% CL on the cross section times branching ratio for single production of (left) $B$ quarks decaying to $b H$ with branching ratios taken from the doublet scenario, and (right) $T$ quarks decaying to $Z t$ with branching ratios taken from the singlet scenario. Figures are from (left) Ref. [12] and (right) Ref. [7].

\section{Conclusions}

The ATLAS Collaboration has an active and broad VLQ search program. The combination of searches for pair-production with $36 \mathrm{fb}^{-1}$ of $p p$ LHC collision data at $\sqrt{s}=13 \mathrm{TeV}$ recorded by 
the ATLAS detector have now set exclusions at 1.03 (1.31) TeV for any considered decay of $B(T)$ VLQs. Searches for singly-produced VLQs have also been performed with $\sqrt{s}=13 \mathrm{TeV}$ data, and have set competitive exclusions on the production cross section. No evidence for VLQs has yet been seen, but the addition of data from 2017-2018 will extend the reach of the VLQ search.

\section{References}

[1] ATLAS Collaboration, The ATLAS Experiment at the CERN Large Hadron Collider, JINST 3 (2008) S08003.

[2] J.A. Aguilar-Saavedra, R. Benbrik, S. Heinemeyer, and M. Perez-Victoria, A handbook of vector-like quarks: mixing and single production, Phys. Rev. D 88 (2013) 094010.

[3] ATLAS Collaboration, Search for pair production of up-type vector-like quarks and for four-top-quark events in final states with multiple b-jets with the ATLAS detector,JHEP 07 (2018) 089.

[4] ATLAS Collaboration, Search for pair production of heavy vector-like quarks decaying to high- $p_{T} W$ bosons and $b$ quarks in the lepton-plus-jets final state in pp collisions at $\sqrt{s}=13 \mathrm{TeV}$ with the ATLAS detector, JHEP 10 (2017) 141.

[5] ATLAS Collaboration, Search for pair production of heavy vector-like quarks decaying into high- $p_{T}$ $W$ bosons and top quarks in the lepton-plus-jets final state in pp collisions at $\sqrt{s}=13 \mathrm{TeV}$ with the ATLAS detector, JHEP 08 (2018) 048.

[6] ATLAS Collaboration, Search for pair production of vector-like top quarks in events with one lepton, jets, and missing transverse momentum in $\sqrt{s}=13 \mathrm{TeV}$ pp collisions with the ATLAS detector, JHEP $\mathbf{0 8}$ (2017) 052.

[7] ATLAS Collaboration, Search for pair-and single-production of vector-like quarks in final states with at least one $Z$ boson decaying into a pair of electrons or muons inpp collision data collected with the ATLAS detector at $\sqrt{s}=13 \mathrm{TeV}$, https://arxiv.org/abs/1806.10555, submitted to Phys. Rev. D, (2018).

[8] ATLAS Collaboration, Search for new phenomena in events with same-charge leptons and b-jets in pp collisions at $\sqrt{s}=13 \mathrm{TeV}$ with the ATLAS detector, https://arxiv.org/abs/1807.11883, submitted to JHEP, (2018).

[9] ATLAS Collaboration, Search for pair production of heavy vector-like quarks decaying into hadronic final states in pp collisions at $\sqrt{s}=13 \mathrm{TeV}$ with the ATLAS detector, https://arxiv.org/abs/1808.01771, submitted to Phys. Rev. D, (2018).

[10] ATLAS Collaboration, Combination of the searches for pair-produced vector-like partners of the third generation quarks at $\sqrt{s}=13 \mathrm{TeV}$ with the ATLAS detector, https://arxiv.org/abs/1808.02343, submitted to Phys. Rev. Lett., (2018).

[11] ATLAS Collaboration, Search for single production of vector-like quarks decaying into Wb in pp collisions at $\sqrt{s}=13 \mathrm{TeV}$ with the ATLAS detector, ATLAS-CONF-2016-072, http://cdsweb.cern.ch/record/2206226, (2016).

[12] ATLAS Collaboration, Search for single production of a vector-like B quark decaying into a bottom quark and a Higgs boson which decays into a pair of photons, ATLAS-CONF-2018-024, http://cdsweb.cern.ch/record/2628759, (2018).

[13] M. Buchkremer, G. Cacciapaglia, A. Deandrea, and L. Panizzi, Model Independent Framework for Searches of Top Partners,Nucl. Phys. B 876 (2013) 376-417.

[14] O. Matsedonskyi, G. Panico, and A. Wulzer, On the Interpretations of Top Partners Searches,JHEP 12 (2014) 097. 